\title{
Breakup of a particulate suspension jet
}

\author{
J. Château and H. Lhuissier* \\ Aix Marseille Université, CNRS, IUSTI, Marseille, France
}

(Received 27 May 2018; revised manuscript received 17 August 2018; published 10 January 2019)

\begin{abstract}
As viscosity is increased, a liquid capillary jet accelerated by gravity stretches over increasingly large distances before eventually breaking up. This Newtonian behavior is profoundly altered for particulate suspensions. Adding solid particles to a liquid, which increases the effective viscosity, can paradoxically shorten the jet considerably [as first reported by Furbank and Morris, Phys. Fluids 16, 1777 (2004)]. This apparent contradiction is rationalized by considering finite-size effects occurring at the scale of a few particles. A model is presented which captures the breakup length of suspension jets observed experimentally for a broad range of liquid viscosities, particle sizes, and extrusion velocities of the jet and recovers the Newtonian case for vanishing particle sizes. These results can be readily extended to any stretched jet configuration and potentially to other fluid media having a granularity.
\end{abstract}

DOI: 10.1103/PhysRevFluids.4.012001

The fragmentation, or atomization, of a liquid involves the formation of transient, stretched, liquid jets, which eventually break up and resolve in drops [1,2]. For homogeneous viscous jets, the rate of pinching is inversely proportional to the liquid viscosity [3] and pinchoff proceeds continuously down to atomic scales [4-6]. Highly viscous jets can therefore reach considerable lengths before breaking up, which explains the seemingly never-breaking threads one can form with honey [7-11] or the notorious difficulty of atomizing extension-thickening polymer solutions [12]. However, if the case of most homogeneous liquids is understood [13,14], that of polyphasic media, such as particulate suspensions, has received much less attention although they are ubiquitous media demanding increasingly smaller processing scales (of, e.g., encapsulation, of printing or molding, and in food, cosmetic, paper, coating, or building industries), for which specific atomization behaviors can be expected. Indeed, as breakup proceeds, their intrinsic granularity is necessarily probed at some point by the vanishing dimension of the jets, and finite-size effects eventually matter. Recent observations on pending drops [15,16] and liquid bridges [17] have reported different facets of adding macroscopic particles to a viscous liquid thread, such as (i) an increase in the thread effective viscosity delaying the breakup and (ii) finite-size effects specific to particulate suspensions hastening the breakup, but the balance between these antagonist effects at the scale of a whole jet is unknown. Therefore, even the basic length scales produced by the fragmentation of a suspension jet, namely, the breakup length and drop sizes, have remained undetermined.

We tackle this fundamental question on the simplest, albeit generic, configuration of a stretched jet: a straight, gravity-stretched jet with a constant flow rate. We consider non-Brownian and rigid particules suspended in a highly viscous Newtonian liquid at a large solid volume fraction (50\%), at which a suspension can flow steadily (without jamming) and shows a pseudo-Newtonian bulk rheology involving contacts between particles [18]. By varying the liquid viscosity, flow rate, and particle size, this experimental system allows us to vary the stretching and onset of finite-size effects,

*henri.lhuissier@univ-amu.fr 

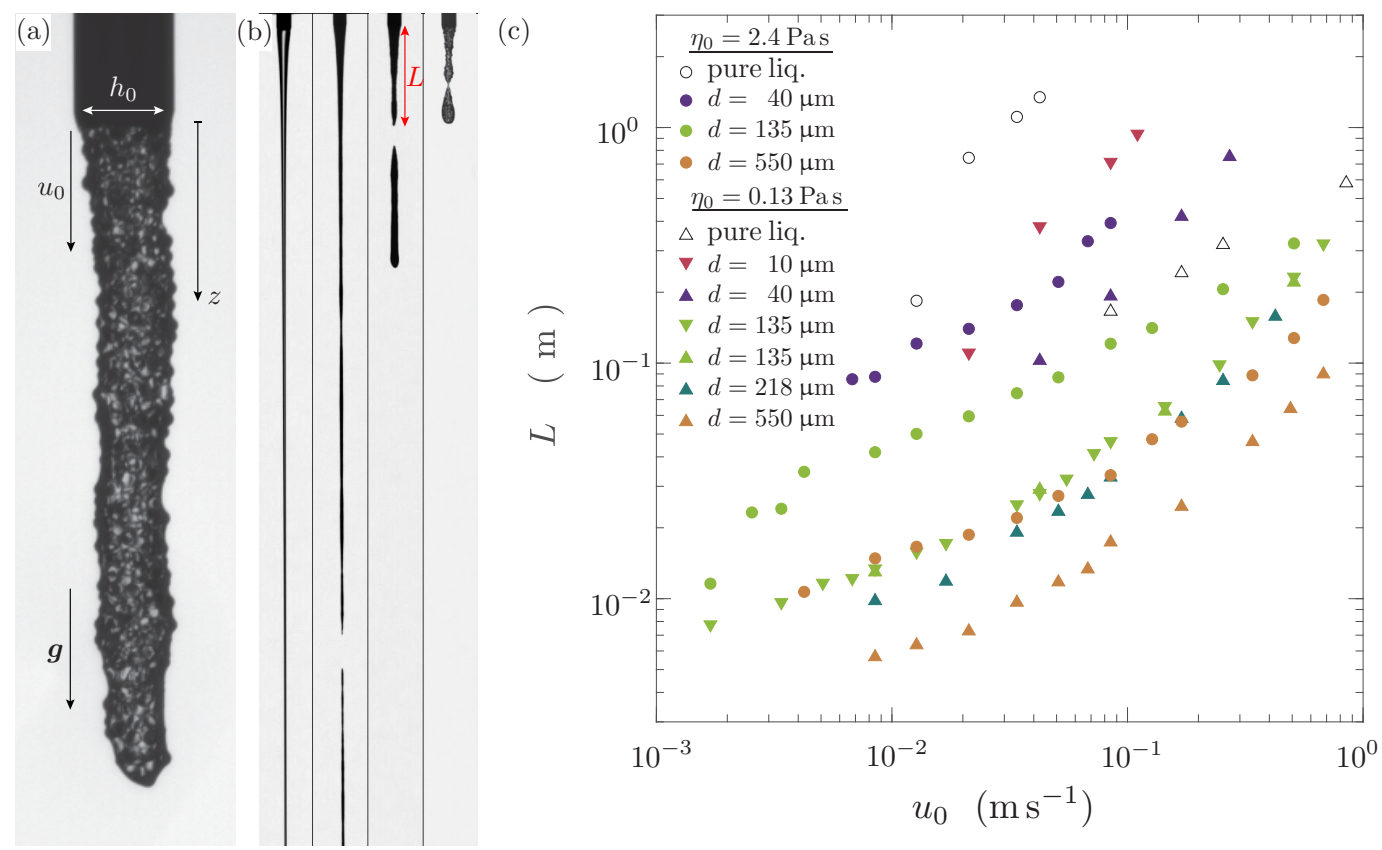

FIG. 1. (a) A particulate suspension jet stretched by gravity ( $d=550 \mu \mathrm{m}, u_{0}=16.8 \mathrm{~cm} / \mathrm{s}$ ). (b) From left to right: Newtonian liquid jet and suspension jets with a particle volume fraction of $50 \%$ and increasing particle diameter $(d=40,135$, and $550 \mu \mathrm{m})$ having the same effective shear viscosity $(\sim 5 \mathrm{Pas})$ and flow rate $\left(u_{0}=4.2 \mathrm{~cm} / \mathrm{s}\right)$. The liquid jet is five times longer than shown. (c) Breakup length vs extrusion velocity for suspensions (• PEGPG, $455 \mathrm{wt} \%$ PEGPG, $\mathbf{\nabla} 30.5 \mathrm{wt} \%$ Ucon oil), and pure liquids (॰ PEGPG, $\triangle 30.5 \mathrm{wt} \%$ Ucon oil).

which is crucial to explore the different breakup regimes and decipher the mechanisms selecting the jet length.

The experiment consists in observing the breakup of a suspension jet stretched by gravity. The jet is formed by extruding the suspension vertically from a large syringe through a small nozzle with inner diameter $h_{0}=2.90 \mathrm{~mm}$ (a cylindrical stainless steel tube with length $10 \mathrm{~mm}$ and thickness $0.35 \mathrm{~mm}$ ). A constant flow rate, with mean velocity at the nozzle of $2 \mathrm{~mm} / \mathrm{s} \lesssim u_{0} \lesssim 1 \mathrm{~m} / \mathrm{s}$, is imposed with a rigid piston and a precision linear motor (M414.2PD, PI). The suspension consists of spherical, polystyrene particles (Dynoseed TS, Microbeads), with density $\rho=1050 \mathrm{~kg} / \mathrm{m}^{3}$, immersed in an isodensity, Newtonian liquid. All the suspensions are prepared at the same particle volume fraction $\phi=50 \%$, which is conserved within $1 \%$ during the extrusion (as verified by weighing $30 \mathrm{~mL}$ of extruded suspension and its dry content). The particle diameter $d$ is varied between 10 and $550 \mu \mathrm{m}$ (for each suspension the relative standard deviation of $d$ is below 8\%). The liquid is either (i) pure PEGPG [3.9 kg/mol poly(ethylene glycol-ran-propylene glycol)-monobutylether by Sigma-Aldrich, with surface tension $\sigma=36 \mathrm{mN} / \mathrm{m}$ and viscosity $\eta_{0}=2.4 \mathrm{~Pa}$.s], (ii) a $45 \mathrm{wt} \%$ aqueous solution of PEGPG $\left(\sigma=38.5 \mathrm{mN} / \mathrm{m}, \eta_{0}=0.13 \mathrm{~Pa} . \mathrm{s}\right)$, or (iii) a $30.5 \mathrm{wt} \%$ aqueous solution of Ucon oil (polyalkylene glycol-composed lubricant Ucon 75-H 90000 by Dow, $\sigma=$ $50.5 \mathrm{mN} / \mathrm{m}, \eta_{0}=0.13 \mathrm{~Pa} . \mathrm{s}$ ). The jet is backlit and imaged with one or two side cameras (with a resolution down to $60 \mu \mathrm{m}$ ). The jet thickness profile and breakup location are obtained by image thresholding. All experiments and rheological measurements are performed at $22 \pm 1{ }^{\circ} \mathrm{C}$.

The profound influence of the particles on the jet breakup is illustrated in Fig. 1(b) (see also the movie in the Supplemental Material [19]). Suspension jets with a particle size increasing from 40 to $550 \mu \mathrm{m}$ are compared to a pure liquid jet having the same flow rate $\left(u_{0}=4.2 \mathrm{~cm} / \mathrm{s}\right)$ and typical effective viscosity ( $\sim 5 \mathrm{~Pa}$ s). Clearly, the suspension jets are more corrugated and fragment 

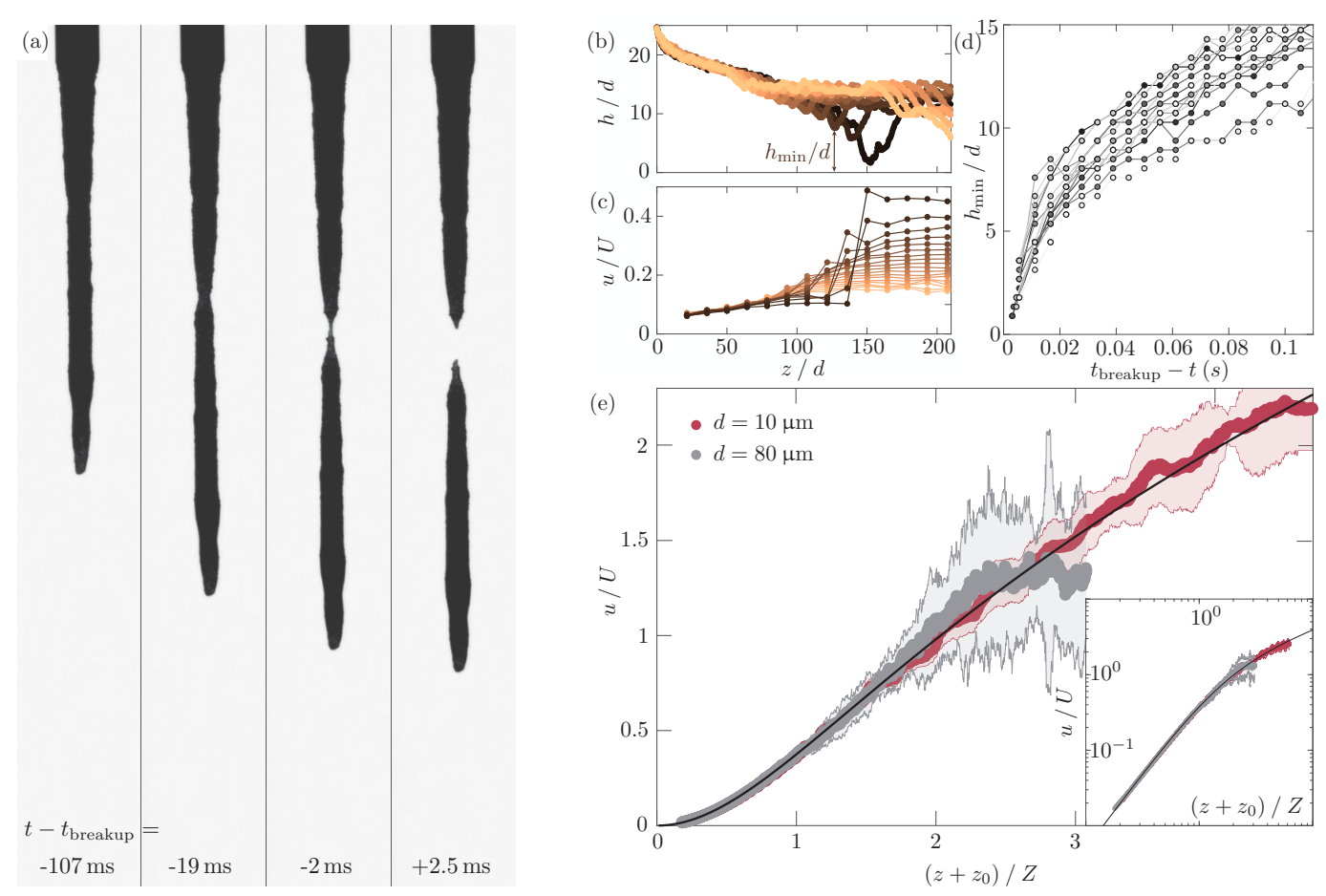

FIG. 2. (a) Localized pinching hastening the breakup of a suspension jet ( $d=135 \mu \mathrm{m}, \eta_{0}=0.13 \mathrm{~Pa}$ s, $u_{0}=4.2 \mathrm{~cm} / \mathrm{s}, h_{0}=2.90 \mathrm{~mm}$, times to breakup are indicated at the bottom). See movies in the Supplemental Material [19]. [(b), (c)] Jet thickness and velocity profiles from breakup to breakup for the sequence shown in panel (a) (the profiles are sampled every $5.6 \mathrm{~ms}$; later profiles are darker). (d) Time evolution of the local minimal diameter until pinchoff for 17 uncorrelated breakup events [same conditions as in panels (a)-(c)]. (e) Steady velocity profile upstream of the breakup for two different particles sizes and a large nozzle, $h_{0}=$ $11.6 \mathrm{~mm}\left(\eta_{0}=0.13 \mathrm{~Pa} \mathrm{~s}\right)$. The solid line is the Newtonian profile (1). $z_{0}$ embeds the extrusion velocity $u_{0}$. The envelop indicates the standard deviation. Inset: Same data in logarithmic scales.

much faster than the liquid jet (the latter is actually five times longer than shown), and, among suspensions, jets with large particles fragment more quickly than those with small particles. To quantify this shortening, we measure the jet length, $L$, which we define as the distance between the nozzle and the most upstream breakup location, while varying systematically $u_{0}, \eta_{0}$, and $d$. The length $L$ fluctuates from breakup to breakup (within $\approx 30 \%$ for a given set of parameters and without strong dependence to the latter) as a consequence of the intrinsically disordered state of the suspensions. In the following, we focus on the mean value obtained by averaging over typically $10^{2}$ breakups.

The raw measurements of $L$ are presented in Fig. 1(c). For a given particle size, the jet length is found to follow the same trends as for a viscous Newtonian liquid. $L$ increases with increasing extrusion velocity, $u_{0}$, and increasing viscosity of the suspending liquid, $\eta_{0}$. However, for fixed $u_{0}$ and $\eta_{0}$, the jet systematically and monotonically shortens with increasing particle size. The shortening is significant, reaching a factor $\sim 10^{2}$ relative to the liquid case for the largest particles $(d=550 \mu \mathrm{m})$, and holds all over the large range of $u_{0}$ and $\eta_{0}$ explored. This has the somehow counterintuitive consequence (observed, e.g., for $d \geqslant 135 \mu \mathrm{m}$ and $\eta_{0}=0.13 \mathrm{~Pa}$ s) that adding particles to a jet at a large volume fraction can shorten the jet in spite of increasing its viscosity, which should delay breakup and lengthen the jet.

The reason for this apparent paradox lies in the dynamics of jet fragmentation. Figure 2(a) presents a typical suspension jet over the few instants preceding its breakup (see also the movie 
in the Supplemental Material [19]). It shows that, as reported for pending drops and capillary bridges [15-17,20-22], the breakup results from a localized necking of the jet, which seems to fail catastrophically. This is confirmed by following, in Figs. 2(b) and 2(c), the evolution of the thickness and velocity profiles along the jet back to the previous pinchoff ( $u$ is measured from the jet surface corrugations displacement by a correlation method (PIV) with a relative precision $\sim 10^{-2}$; see Ref. [23]). Over the period between two successive breakups, the deformation of the jet changes qualitatively. Initially, the jet diameter, $h$, and stretching rate, $\partial_{z} u$, are uniform over long distances. Later, however, a neck develops where the pinching localizes and accelerates, as attested by increasingly large and localized stretching rates. In agreement with previous experiments with systematically varied particle sizes and volume fractions [22], this catastrophic acceleration of the pinching is found to occur for $h=n d \approx 10 d$, i.e., when the jet diameter has thinned down to a few particles sizes. The $d$ scaling is a signature of the finite-size effects involved in the pinching acceleration, whereas $n$ is a nontrivial function of $\phi$, which has also been measured on capillary bridges to be close to 10 for $\phi=50 \%$ [22].

The observations above suggest that at least two facets of the deformation of a suspension jet need to be considered to rationalize its shortness: (i) the stretching base state of a smooth unmodulated jet and (ii) deviations from this base state leading to a finite-time pinchoff. Clearly, both facets are impacted by the presence of the particles, since the latter increases the effective viscosity of the jet [18] and triggers the necking, hastening the breakup. Nevertheless, they also share similarities with the Newtonian case, as attested by the convergence of the behaviors when the particle size is decreased. We thus consider, first, the steady base state of a Newtonian jet with viscosity $\eta$ and density $\rho$ (neglecting, for now, the Plateau-Rayleigh capillary destabilization). For sufficiently long jets $\left(L \gg h_{0}\right)$, this base state is well captured by a unidimensional, slender-slope approximation of the momentum and mass equations,

$$
u u_{z}=g+\frac{3 \eta}{\rho} \frac{\left(h^{2} u_{z}\right)_{z}}{h^{2}}, \quad h^{2}=\frac{h_{0}^{2} u_{0}}{u},
$$

which naturally introduces the viscogravity length and velocity scales, $Z=\left(9 \eta^{2} / \rho^{2} g\right)^{1 / 3}$ and $U=(3 \eta g / \rho)^{1 / 3}$, respectively [24-26]. At short distance from the nozzle $(u \ll U)$, the stretching is limited by viscosity and $u / U \simeq(z / Z)^{2} / 2$. Conversely, at long distance $(u \gg U)$, the jet is free falling and $u / U \simeq \sqrt{2 z / Z}$.

Using these stretching laws for a suspension jet requires that a Newtonian extensional effective viscosity can be defined and measured. We validate this point (already adopted in previous works [15-17]) by performing velocity measurements with a larger nozzle $\left(h_{0}=11.6 \mathrm{~mm}\right.$ and $\eta_{0}=0.13 \mathrm{~Pa}$ s) in cases when the rheological signature on the stretching base state and deviations from this base state are decoupled, i.e., when $L \gg Z$. The velocity $u$ is measured from the thickness profile assuming a steady jet, i.e., $u=u_{0} h_{0}^{2} / h^{2}$. Surface PIV measurements (see Ref. [23]) yield the same values within 5\%, which confirms the steadiness. As seen from Fig. 2(e), the experimental velocity profiles obtained for two different particle sizes follow closely the Newtonian profile (1) upstream from the breakup. This collapse validates the Newtonian effective viscosity approach. It also yields a measurement of the effective extensional viscosity, $\rho \sqrt{g Z^{3}} / 3$, from the fitting length $Z$. In agreement with recent experiments and simulations [22,27,28], the extensional measurements $\left(\eta / \eta_{0} \simeq 54\right.$ and $52 \pm 2$ for $d=80$ and $10 \mu \mathrm{m}$, respectively) closely agree with the relative shear effective viscosity $\left(\eta / \eta_{0} \simeq 52\right.$ and $42 \pm 8$, respectively), which is measured, independently, in a dedicated shear cell (see Ref. [29]) and is itself consistent with shear viscosity values reported in the literature for $\phi=50 \%$ [18].

On the grounds of the validity of the Newtonian base state for a "thick" jet and of the heuristic onset for the finite-size catastrophic breakup, we now propose a model for the jet length. Assuming that the Newtonian approach is valid until finite-size effects come into play and that the subsequent pinch-off duration is negligible, one obtains

$$
h(z=L)=n d,
$$



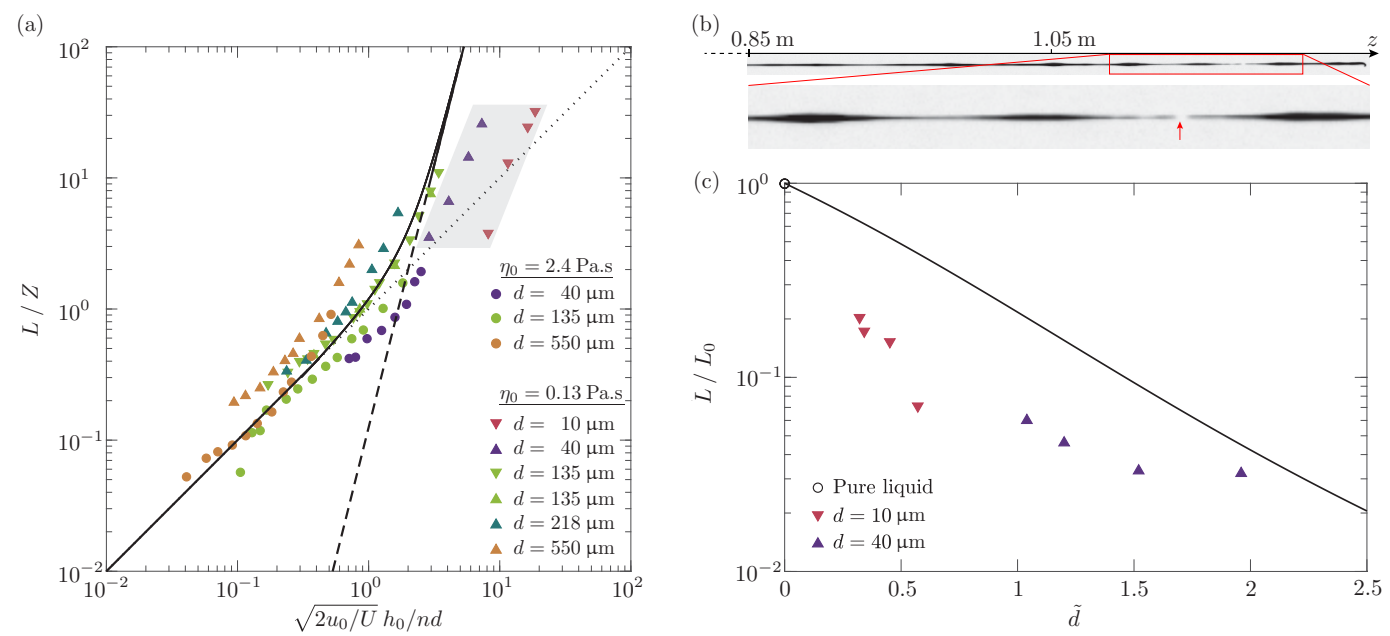

FIG. 3. (a) Dimensionless breakup length vs extrusion velocity for the suspensions [same data as in Fig. 1(c)]. The dashed and dotted lines are, respectively, the predictions (3) and (4) for the viscous and inertial stretching regimes, with $n=10$. The solid line is the numerical solution (obtained with a relative precision smaller than $10^{-3}$ using a standard integration routine of MATHEMATICA) of Eqs. (1) and (2) connecting both asymptotes. The gray domain encloses the cases developing a capillary modulation (see text). (b) Jet with small particles $(d=10 \mu \mathrm{m})$ developing a Plateau-Rayleigh modulation prior to breaking up (at the arrow). (c) Breakup length in the presence of a capillary modulation of the jet vs $\tilde{d}=\left(2 U^{2} L_{0} / u_{0}^{2}\right)^{1 / 4} n d / h_{0}$. The data are those inside the gray domain in panel (a). The solid line is Eq. (6) with $n=10$. The value of $\alpha(=30)$ is calibrated with a pure liquid $\left(\eta_{0}=2.4 \mathrm{~Pa} \mathrm{~s}\right)$.

with $h(z)$ defined by Eq. (1). For short jets, this condition yields

$$
\frac{L}{Z}=\sqrt{\frac{2 u_{0}}{U}} \frac{h_{0}}{n d},
$$

whereas for long jets it gives

$$
\frac{L}{Z}=\frac{1}{2}\left(\frac{u_{0}}{U}\right)^{2}\left(\frac{h_{0}}{n d}\right)^{4} .
$$

These predictions for the viscous and the free-fall stretching regimes are expected to apply for $\sqrt{2 u_{0}} h_{0} / \sqrt{U} n d \ll 1$ and $\sqrt{2 u_{0}} h_{0} / \sqrt{U} n d \gg 1$, respectively. Consistently with the neglect of the Plateau-Rayleigh instability and of the terminal capillary pinchoff, they do not involve surface tension. They are compared in Fig. 3(a) to the experimental data, without adjustable parameter. In spite of their simplifications, they capture both the magnitude and the dependence of $L$ on $\sqrt{2 u_{0}} h_{0} / \sqrt{U} n d$ for all of the measurements (spanning ranges of $\eta_{0}, d$, and $u_{0}$ of respectively 18 , 55 , and 500), except for the longest jets $(L / Z \gg 1)$ with the smallest particles $(d=10$ and $40 \mu \mathrm{m})$, which are highlighted in gray. The latter data precisely concern the case when the Plateau-Rayleigh capillary instability of the jet cannot be neglected any more, as direct observation confirms. Indeed, as illustrated in Fig. 3(b) for $d=10 \mu \mathrm{m}$, and by contrast with the case of larger particles [see Fig. 2(a)], the jet develops periodic diameter modulations of a capillary origin (as confirmed by the value of the selected wavelength experimentally observed; see Ref. [30]), prior to breaking up precisely at one modulation neck.

To rationalize the experimental breakup length for these "small" particles, we consider that the condition for the catastrophic breakup of the jet does not apply to the unmodulated base state (1), but rather at the neck of a capillary modulation developing on this base state, where the diameter is 
locally minimal. In this framework, the breakup length verifies

$$
\int_{0}^{L} u^{-1} d z=\alpha \eta[h(L)-n d] / \sigma,
$$

expressing that the travel time down to $L$ equals the time to develop a diameter modulation of order $h(L)-n d$ at the typical viscosity-limited growth rate of the Plateau-Rayleigh instability, $\sigma / \eta h(L)$ [31], which is relevant here since $\eta / \sqrt{\rho \sigma h(L)} \gg 1 . \alpha$ is a prefactor of order 10, embedding both the instantaneous growth rate prefactors and those due to the amplitude gain history along the stretched jet $[8,10,11]$, which we calibrate with a pure liquid (see below). For small particles, the breakup occurs in the free-fall regime $(L \gg Z$ ). We therefore use Eq. (5) with the free-fall limit of Eq. (1) and obtain the following implicit equation for $L$ :

$$
L / L_{0}=\left[1-\tilde{d}\left(L / L_{0}\right)^{1 / 4}\right]^{4 / 3},
$$

where

$$
L_{0}=\frac{1}{2}\left(\frac{\alpha \eta \sqrt{U u_{0}}}{\sigma} \frac{h_{0}}{Z}\right)^{4 / 3} Z
$$

is the length for a vanishing particle diameter, i.e., that expected for a pure liquid having the same effective viscosity, and

$$
\tilde{d}=\left(\frac{2 U^{2} L_{0}}{u_{0}^{2} Z}\right)^{1 / 4} \frac{n d}{h_{0}}
$$

is the dimensionless particle size controlling the shortening of the jet relative to $L_{0}$. Note that we recover for $L_{0}$ the expression derived in Ref. [10] for a Newtonian liquid when the jet base state stretching is independent of surface tension. Equation (6) is compared, without adjustable parameter, to those measurements of long jets with small particles that develop capillary modulations in Fig. 3(b) (the prefactor $\alpha=30$ is calibrated independently from experiments with a pure liquid of similar viscosity, $\eta_{0}=2.4 \mathrm{~Pa} \mathrm{~s}$, for which $L_{0}=L$ ). It is found to capture both the magnitude and the trend of the breakup length with the dimensionless particle diameter $\tilde{d}$. Note that Eq. (6) actually contains the limit without capillarity discussed previously. Indeed, for large particles it becomes asymptotically $L / L_{0}=\tilde{d}^{-4}$, which is exactly Eq. (4). Therefore, $\tilde{d} \sim 1$ represents the boundary between the regime when the jet length is determined by the sole particle size $(\tilde{d} \gg 1)$ and the regime when both finite-size effects and capillarity matter $(\tilde{d} \ll 1)$.

To conclude, the fragmentation of a thick suspension jet eventually probes the suspension granularity and thus naturally involves both the bulk rheology controlling the initial stretching and destabilization, and finite-size effects triggering a localized acceleration of the pinching akin to a catastrophic failure. For a concentrated suspension, these two stages are essentially decoupled and have separated timescales, which permits us to understand the breakup length of a stretched jet in the different regimes where viscosity, inertia, or capillary destabilization are involved. This also rationalizes why adding particles to a jet at a large volume fraction can shorten the jet in spite of increasing its viscosity. This scenario quantitatively explains the experimental jet lengths for the large solid volume fraction $\phi(=50 \%)$ considered here. It is also expected to apply to other values of $\phi$, provided the effective Newtonian stretching rate $\left(\propto \eta^{-1}\right)$ remains much smaller than that of the last instants of the pinchoff $\left(\propto \eta_{0}^{-1}\right)$ [16,17], i.e., provided the suspension is concentrated enough. In such case, the model offers a direct prediction for the jet length in terms of $\phi$ from the $\phi$ dependance of the effective viscosity, $\eta / \eta_{0}$, and of the onset of the finite-size effects, $n$ [18,22]. Besides, these results are readily extendable to any stretched jet configuration and should therefore apply to most atomization processes. They also appeal for further efforts to understand the size of the drops, or fragments, as well as to be extended to other polyphasic media, such as foams, emulsions, or fiber suspensions. 
This work has been financially supported by the Agence Nationale de la Recherche through Grant No. ANR-14-ACHN-0019-01.

[1] F. Savart, Mémoire sur la constitution des Veines liquides lancées par des orifices circulaires en mince paroi, Ann. Chim. Phys. France 53, 337 (1833).

[2] J. Plateau, Statique Expérimentale et Théorique Des Liquides Soumis Aux Seules Forces Moléculaires (Ghauthier-Villard, Paris, 1873).

[3] J. Lord Rayleigh, On the instability of a cylinder of viscous liquid under capillary forces, Phil. Mag. 34, 145 (1892).

[4] J. Eggers, Universal Pinching of 3D Axisymmetric Free Surface Flow, Phys. Rev. Lett. 71, 3458 (1993).

[5] D. Papageorgiou, On the breakup of viscous liquid threads, Phys. Fluids 7, 1529 (1995).

[6] A. Rothert, R. Richter, and I. Rehberg, Transition from Symmetric to Asymmetric Scaling Function Before Drop Pinch-Off, Phys. Rev. Lett. 87, 084501 (2001).

[7] B. Ambravaneswaran, H. Subramani, S. Phillips, and O. Basaran, Dripping-Jetting Transitions in a Dripping Faucet, Phys. Rev. Lett. 93, 034501 (2004).

[8] U. Sauter and H. Buggish, Stability of initially slow viscous jets driven by gravity, J. Fluid Mech. 533, 237 (2005).

[9] S. Senchenko and T. Bohr, Shape and stability of a viscous thread, Phys. Rev. E 71, 056301 (2005).

[10] A. Javadi, J. Eggers, D. Bonn, M. Habibi, and N. Ribe, Delayed Capillary Breakup of Falling Viscous Jets, Phys. Rev. Lett. 110, 144501 (2013).

[11] S. Le Dizès and E. Villermaux, Capillary jet breakup by noise amplification, J. Fluid Mech. 810, 281 (2016).

[12] G. McKinley, Visco-elasto-capillary thinning and breakup of complex fluids, in Rheology Reviews, edited by D. Binding and K. Walters (British Society of Rheology, Aberystwyth, 2005), pp. 1-48.

[13] R. Suryo and O. Basaran, Local dynamics during pinch-off of liquid threads of power law fluids: Scaling analysis and self-similarity, J. Non-Newtonian Fluid Mech. 138, 134 (2006).

[14] J. Eggers and E. Villermaux, Physics of liquid jets, Rep. Prog. Phys. 71, 036601 (2008).

[15] R. Furbank and J. Morris, An experimental study of particle effects on drop formation, Phys. Fluids 16, 1777 (2004).

[16] C. Bonnoit, T. Bertrand, E. Clément, and A. Lindner, Accelerated drop detachment in granular suspensions, Phys. Fluids 24, 043304 (2012).

[17] W. Mathues, C. McIlroy, O. Harlen, and C. Clasen, Capillary breakup of suspensions near pinch-off, Phys. Fluids 27, 093301 (2015).

[18] É. Guazzelli and O. Pouliquen, Rheology of dense granular suspensions, J. Fluid Mech. 852, 35 (2018).

[19] See Supplemental Material at http://link.aps.org/supplemental/10.1103/PhysRevFluids.4.012001 for the movies of the sequence shown in Figs. 1(b) and 2(a).

[20] M. Miskin and H. Jaeger, Droplet formation and scaling in dense suspensions, Proc. Natl. Acad. Sci. USA 109, 4389 (2012).

[21] Z. Pan, N. Louvet, Y. Hennequin, H. Kellay, and D. Bonn, Drop formation in shear-thickening granular suspensions, Phys. Rev. E 92, 052203 (2015).

[22] J. Château, É. Guazzelli, and H. Lhuissier, Pinch-off of a viscous suspension thread, J. Fluid Mech. 852, 178 (2018).

[23] The surface velocity of the jet is measured locally from the axial displacement of the jet surface corrugation pattern caused by the particles. A vector encoding the axial corrugation pattern, as observed from the camera's viewpoint, is obtained by averaging the image intensity over each vertical pixel line. Conventional particle image velocimetry routines of subvector correlation between successive frames and subpixel interpolation yield the displacement $\left(\sim 10^{2}\right.$ pixels $)$ of each jet portion.

[24] D. Brown, A study of the behavior of a thin sheet of moving liquid, J. Fluid Mech. 10, 297 (1961).

[25] N. Clarke, The asymptotic effects of surface tension and viscosity on an axially-symetric free jet of liquid under gravity, Quart. Journ. Mech. Appl. Math 22, 247 (1969). 
[26] J. Eggers and T. Dupont, Drop formation in a one-dimensional approximation of the Navier-Stokes equation, J. Fluid Mech. 262, 205 (1994).

[27] R. Seto, G. Giusteri, and A. Martiniello, Microstructure and thickening of dense suspensions under extensional and shear flows, J. Fluid Mech. 825, R3 (2017).

[28] O. Cheal and C. Ness, Rheology of dense granular suspensions under extensional flow, J. Rheol. 62, 501 (2018).

[29] The shear viscosity is measured in a dedicated plate/plate device allowing a large cell gap $(e>60 d)$, which consists of a rotating disk (Anton Paar MCR 501, with radius $R=25 \mathrm{~mm}$ and rotation rate $\omega$ ) immersed inside a cylindrical container (with radius $30 \mathrm{~mm}$ ) at a distance $e$ from the flat bottom of the container. It is calibrated with two Newtonian liquids giving the same calibration. The shear viscosity of the suspensions is measured over the same range of deformation rates, $1 \mathrm{~s}^{-1} \leqslant 3 \pi R \omega / 2 e \leqslant 10 \mathrm{~s}^{-1}$, as in the jet $\left(1 \mathrm{~s}^{-1} \lesssim \partial_{z} u \lesssim 10 \mathrm{~s}^{-1}\right)$. The measurements are time independent, shear rate independent (within $10 \%$ ), and gap independent (within $5 \%$ between $e=5 \mathrm{~mm}$ and $9 \mathrm{~mm}$ ).

[30] The wave number observed in Fig. $3(\mathrm{~b}), k h \approx 0.10-0.15(2 \pi / k \approx 6 \mathrm{~cm}$ and $h \approx 1-1.5 \mathrm{~mm})$, agrees with the most unstable mode of the Plateau-Rayleigh instability of a cylindrical thread, $k_{\max } h=$ $\sqrt{2 /(1+3 \mathrm{Oh})} \approx 0.15-0.16$, expected from slender slope theory for the large local Ohnesorge number of the jet $(\mathrm{Oh}=\eta / \sqrt{\rho \sigma h} \approx 25-30)[14,31]$. For $u_{0} \gg U$, corrections due to the stretching of the modes along the jet are of order $1[10,11]$.

[31] S. Chandrasekhar, Hydrodynamic and Hydromagnetic Stability (Clarendon Press, Oxford, UK, 1961). 